\title{
AVALIACÃO DA PLACA VERTEBRAL TERMINAL NA COLUNA CERVICAL EM DIFERENTES FAIXAS ETÁRIAS E SUA CORRELAÇÃO COM A ESPESSURA DO DISCO INTERVERTEBRAL
}

\author{
EVALUATION OF TERMINAL VERTEBRAL PLATE ON CERVICAL SPINE AT DIFFERENT \\ AGE GROUPS AND ITS CORRELATION WITH INTERVERTEBRAL DISC THICKNESS
}

\author{
Juliano Silveira Luiz Vieira', \\ Carlos Fernando Pereira da Silva Herrero', \\ Maximiliano Aguiar Porto², \\ Marcello Henrique Nogueira Barbosa ${ }^{3}$, \\ Sérgio Britto Garcia ${ }^{4}$, \\ Leandra Náira Zambelli Ramalho ${ }^{5}$, \\ Helton Luiz Aparecido Defino'
}

\section{RESUMO}

Objetivo: Avaliar por meio de histomorfometria, a espessura da placa vertebral terminal, a espessura do disco intervertebral e a sua correlação nas diferentes faixas etárias, tentando identificar a sua correlação. Métodos: Foram avaliados os segmentos cervicais C4-C5 e C5-C6 de 50 cadáveres humanos, de ambos os sexos, divididos em 5 grupos com intervalo de faixa etária de 10 anos, a partir dos 21 anos de idade. A avaliação da espessura da PVT e do disco intervertebral foi realizada por meio de avaliação histomorfométrica dos cortes histológicos corados pela hematoxilina e eosina. A espessura da PVT inferior de C4, PVT superior de C5, PVT inferior de C5 e PVT superior de C6 foram comparadas entre si e também com a espessura do disco intervertebral interposto entre as respectivas PVT. Resultados: A espessura das placas vertebrais terminais adjacentes ao mesmo DI não apresentou diferença estatística. No entanto, a comparação da espessura das placas vertebrais superior e inferior da mesma vértebra cervical (C5), apresentou diferença estatística em todas as faixas etárias avaliadas. Foi observado coeficiente de correlação estatística, maior que $80 \%$, entre a espessura da

\section{ABSTRACT}

Objective: To evaluate, by means of histomorphometry, terminal vertebral plate thickness, intervertebral disc thickness and its correlation on different age groups, seeking to identify its correlation. Methods: C4-C5 and C5-C6 cervical segments removed from human cadavers of both genders were assessed and divided into five groups of 10-year age intervals, from 21 years old. TVP and intervertebral disc thickness evaluation was made by means of histomorphometry of histological slides stained with hematoxylin and eosyn. Lower C4 TVP, upper C5 TVP, and upper C6 TVP de were compared between each other and to the interposed intervertebral disc thickness between relevant TVP. Results: The thickness of terminal vertebral plates adjacent to the same ID did not show statistic differences. However, the comparison of upper and lower vertebral plates thickness on the same cervical vertebra (C5), showed statistical difference on all age groups studied. We found a statistical correlation coefficient above $80 \%$ between terminal vertebral plate and adjacent intervertebral disc, with

1 - Aluno do Programa de Pós-Graduação da Área Ortopedia, Traumatologia e Reabilitação do Departamento de Biomecânica, Medicina e Reabilitação do Aparelho Locomotor da Faculdade de Medicina de Ribeirão Preto da Universidade de São Paulo.

2 - Médico Residente em Cirurgia da Coluna no Hospital das Clínicas da Faculdade de Medicina de Ribeirão Preto da Universidade de São Paulo.

3 - Professor da Divisão de Radiologia do Departamento de Clínica Médica da Faculdade de Medicina de Ribeirão Preto da Universidade de São Paulo.

4 - Doutor, Professor Associado e Chefe do Departamento de Patologia da Faculdade de Medicina de Ribeirão Preto da Universidade de São Paulo.

5 - Doutora, Docente do Departamento de Patologia da Faculdade de Medicina de Ribeirão Preto da Universidade de São Paulo.

6 - Doutor, Professor Titular Departamento de Biomecânica, Medicina e Reabilitação do Aparelho Locomotor da Faculdade de Medicina de Ribeirão Preto da Universidade de São Paulo.

Trabalho realizado no Departamento de Biomecânica, Medicina e Reabilitação do Aparelho Locomotor da Faculdade de Medicina de Ribeirão Preto da Universidade de São Paulo. Correspondência: Helton L. A. Defino, Departamento de Biomecânica, Medicina e Reabilitação do Aparelho Locomotor da Faculdade de Medicina de Ribeirão Preto da Universidade de São Paulo - USP - Ribeirão Preto (SP), Avenida Bandeirantes, 3900, 11 andar - 14048-900. E-mail: hladefin@fmrp.usp.br 
placa vertebral terminal e o disco intervertebral adjacente, com proporcional redução de espessura de ambas estruturas, nos diferentes níveis cervicais avaliados, e também nas diferentes faixas etárias avaliadas. Conclusão: A placa vertebral terminal apresenta correlação morfológica com o disco intervertebral ao qual entra em contato, e não apresenta correlação com a placa vertebral terminal da mesma vértebra.

Descritores - Coluna vertebral; Disco intervertebral; Cadáver a proportional thickness reduction of both structures on the different cervical levels studied, and also on the different age groups assessed. Conclusion: Terminal vertebral plate shows a morphologic correlation with the intervertebral disc next to $i$, and does not show correlation with the terminal vertebral plate on the same vertebra.

Keywords - Spine; Intervertebral disc; Cadaver

\section{INTRODUÇÃO}

O disco intervertebral (DI) é estrutura avascular e composta de fibras colágenas, água e proteoglicanos ${ }^{(1)}$. A nutrição do disco intervertebral ocorre por meio de difusão dos nutrientes através da placa vertebral terminal ${ }^{(2)}$.

A placa vertebral terminal (PVT) é estrutura anatômica presente nas vértebras e está em íntima relação com o disco intervertebral (DI). A PVT é formada por uma fina camada cartilaginosa localizada cranial e caudalmente entre o DI e o osso esponjoso do corpo vertebral. A sua espessura, em indivíduos adultos, varia de $0,6 \mathrm{~mm}$ a $3 \mathrm{~mm}$. A PVT é constituída por um gel hidratado de moléculas de proteoglicanos que se encontram entrelaçadas por fibras de colágeno ${ }^{(3)}$. A PVT apresenta estrutura semelhante ao DI, não apresentando conexões diretas com o corpo vertebral e conectando-se diretamente ao DI através da lamela média do anulo fibroso ${ }^{(4)}$. A composição bioquímica da PVT é crítica para a manutenção da nutrição e integridade do DI. Os proteoglicanos são os principais reguladores do transporte de solutos essenciais para o interior e fora do $\mathrm{DI}^{(5)}$. Sendo o DI uma estrutura avascular, a sua manutenção depende da difusão dos nutrientes através da PVT. Além de constituir-se como barreira de controle seletivo da passagem de nutrientes para o disco vertebral, a PVT previne a diminuição da concentração de proteoglicanos do material discal intervertebral, fator este crucial na fisiopatologia da doença degenerativa discal ${ }^{(6)}$. A PVT apresenta também importante função mecânica. A pressão hidrostática absorvida pelos discos intervertebrais durante a transmissão axial de cargas é distribuída parcialmente também na PVT, evitando-se assim a excessiva carga sobre o corpo vertebral adjacente ${ }^{(7)}$. Adicionalmente, a PVT atua também como barreira física intermediária, evitando o colapso do disco intervertebral contra o corpo vertebral, durante a distribuição homogênea de cargas hidrostáticas.
Considerando a relação funcional da PVT e do disco intervertebral, foi delineado esse estudo, cujo objetivo foi observar a espessura da PVT, a espessura do disco intervertebral, nas diferentes faixas etárias, tentando identificar a sua correlação.

\section{MÉTODOS}

O trabalho foi aprovado pelo Comitê de Ética Médica do Hospital das Clínicas da Faculdade de Medicina de Ribeirão Preto - USP e pelo Responsável pelo Serviço de Verificação de Óbitos de Ribeirão Preto (SVOI) e do Centro de Medicina Legal (CEMEL).

O material estudado foi retirado durante a autópsia de 50 cadáveres humanos ( 30 do sexo masculino e 20 do sexo feminino), com idade variando de 21 a 69 anos $(45,04 \pm 15,63)$. Os 50 cadáveres foram divididos em cinco grupos etários sendo cada grupo constituído por 10 cadáveres. $\mathrm{O}$ grupo 1 era formado por cadáveres com idade entre 21 e 30 anos, o grupo 2 com idade entre 31 e 40 anos, o grupo 3 com idade entre 41 e 50 anos, o grupo 4 com idade entre 51 e 60 anos e o grupo 5 com idade entre 61 e 70 anos.

Os segmentos vertebrais escolhidos para o estudo foram: C4-C5 e C5-C6, considerando-se o seu grau de mobilidade e a incidência da doença degenerativa discal nesses segmentos (PTGDI).

Os segmentos vertebrais escolhidos para o estudo foram retirados durante autópsia por meio de corte transversal no terço inferior e superior dos corpos vertebrais adjacentes ao disco intervertebral selecionado para o estudo. A seguir foi realizado corte no plano sagital dividindo a peça anatômica ao meio. As duas metades foram seccionadas por meio de corte parassagital com $3 \mathrm{~mm}$ de espessura, tendo sido utilizadas as partes mediais de cada metade para o estudo (Figura 1).

Antes do preparo das peças anatômicas eram realizadas radiografias em ântero-posterior (AP) e perfil, de 

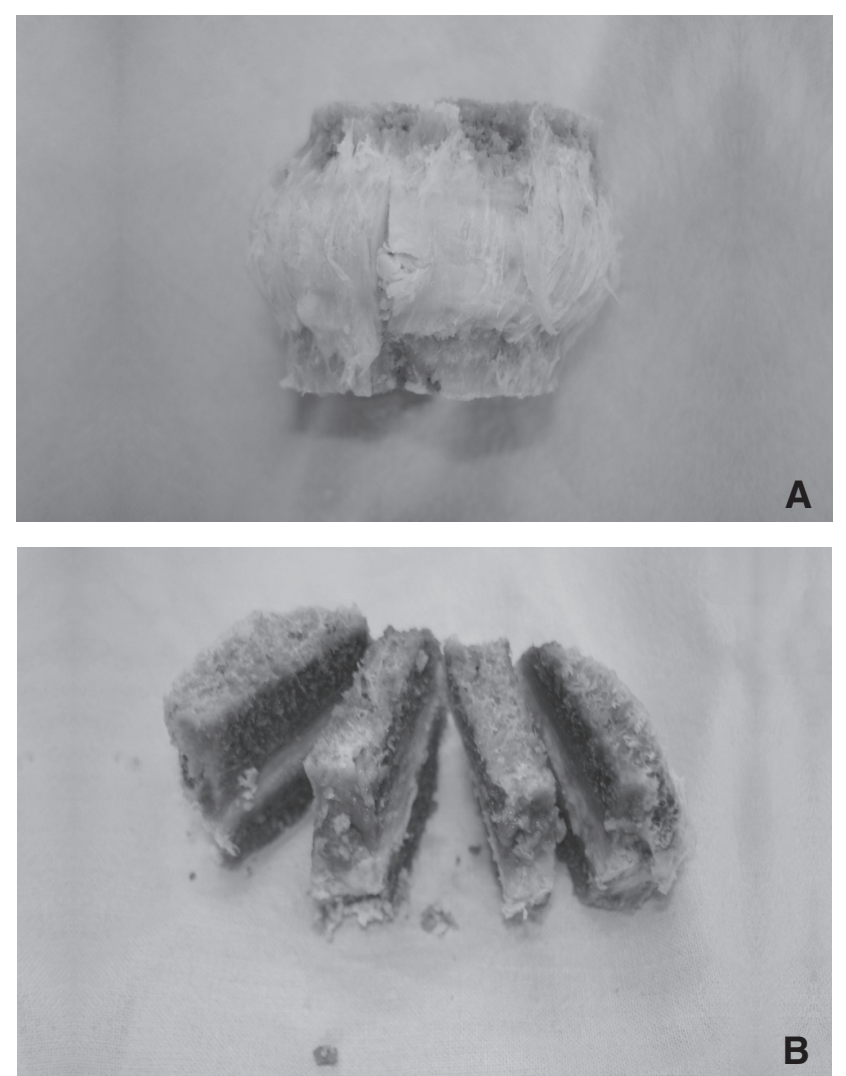

Figura 1 - Vista frontal da peça anatômica contendo o disco intervertebral C5-C6 e parte dos corpos vertebrais adjacentes antes (A) e após (B) a realização dos cortes no plano sagital.

acordo com a técnica padrão para observar a presença de degeneração discal. Foram excluídos do estudo os segmentos que apresentavam sinais radiográficos de degeneração discal. A avaliação das radiografias foi independentemente realizada por médico radiologista. Os parâmetros utilizados para a avaliação da presença de degeneração discal foram: redução do espaço discal, esclerose óssea subcondral, presença de osteofitose vertebral, presença de cistos ósseos de reabsorção subcondral e presença de osteopenia perilesional

Os dois cortes de $3 \mathrm{~mm}$ de espessura, resultantes dos cortes sagital e parassagital da peça anatômica foram preparados para estudo histológico tendo sido fixados em formol neutro a $10 \%$, descalcificados com ácido tricloroacético, e incluídos em parafina. Foram realizados cortes de $5 \mu$ de espessura no eixo sagital das peças anatômicas, que abrangiam os corpos vertebrais adjacentes e o disco intervertebral interposto. Os cortes foram corados pela hematoxilina e eosina e analisados sob microscopia de luz (Figura 2).

A espessura do DI e das PVT adjacentes - PVT distal da vértebra superior e PVT proximal da vértebra inferior - foram avaliadas por meio de técnica de histomorfo-
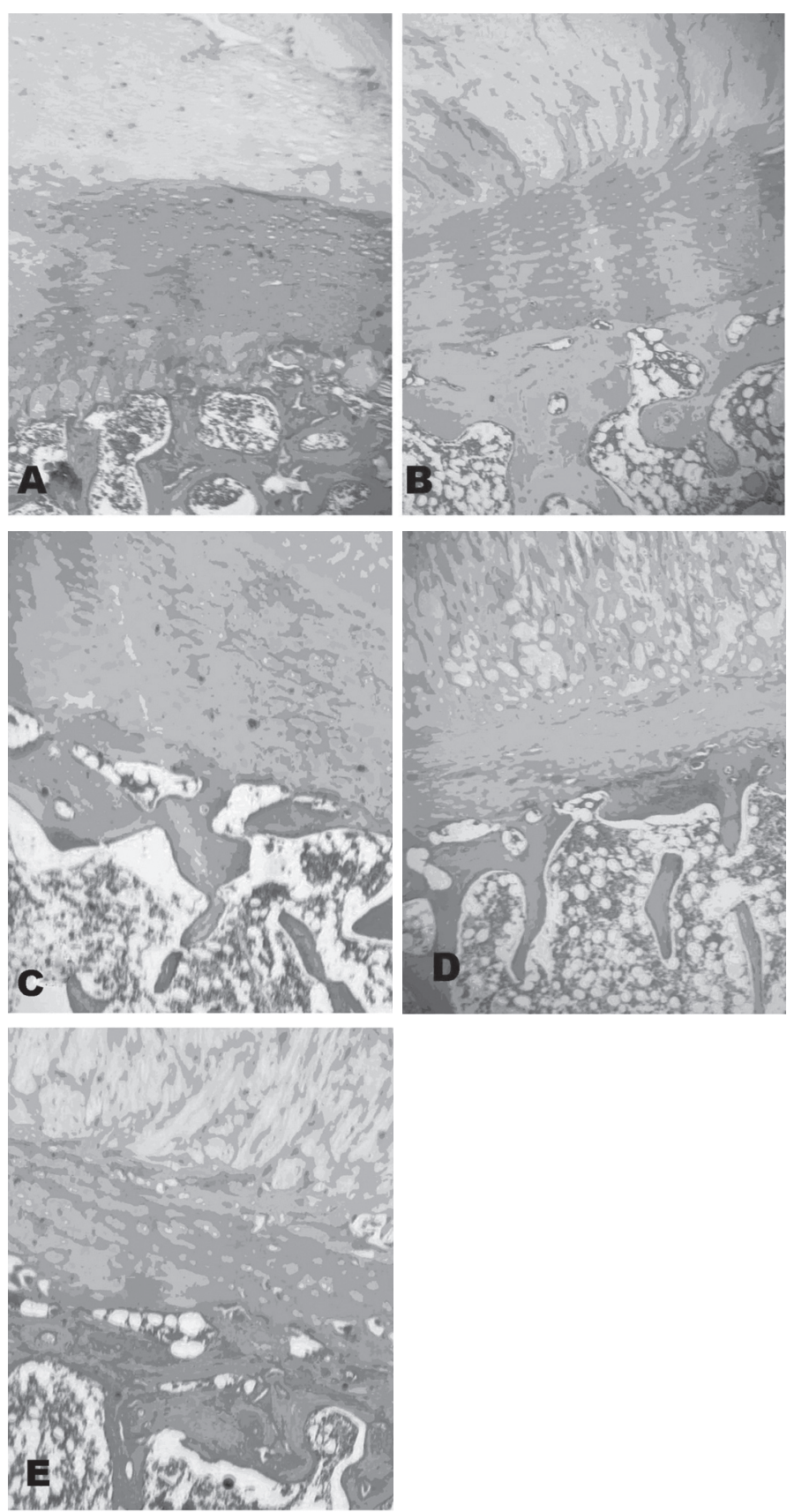

Figura 2 - Fotomicrografia ilustrando a PVT, o disco intervertebral e o osso esponjoso do corpo vertebral , no nível vertebral C4-C5, em indivíduo do grupo 1 (A), grupo 2 (B), grupo 3 (C), grupo 4 (D) e grupo $5(E)$, sob coloração HE e sob aumento de 400 vezes.

metria com utilização de ocular integradora Zeiss ${ }^{\circledR}$ sob aumento de 10 vezes e com objetiva de 40 vezes. O terço central da PVT e do DI foi selecionado para a mensuração da espessura. Foi estudada a relação entre a altura do DI e das placas vertebrais adjacentes na região delimitada para o estudo. Esta correlação foi avaliada no grupo total dos indivíduos estudados e nas diferentes faixas etárias. Foi também avaliada a espessura das estruturas analisadas em relação com o aumento da faixa etária. 
O estudo estatístico dos parâmetros relacionados foi realizado por meio da utilização da análise de variância (ANOVA), utilizando-se o procedimento "PROC GLM do software SAS versão 9".

A correlação entre a espessura da placa vertebral terminal e disco intervertebral nas diferentes faixas etárias foi realizada por meio da metodologia utilizada para a análise de variância tipo "ANOVA", utilizando-se o procedimento " PROC GLM do software SAS versão 9".

\section{RESULTADOS}

Os valores da espessura da PVT e do DI nos segmentos cervicais selecionados para o estudo, estão representados na tabela 1 .

A altura do disco intervertebral C4-C5 e C5-C6 apresentou redução dos valores com o aumento da idade, tendo sido observado diferença estatística significativa entre as faixas etárias estudadas $(\mathrm{p}<0,05)$ (Gráfico 1).
A espessura da PVT também apresentou redução dos valores com o aumento da idade, tendo sido observado diferença estatística significante $(\mathrm{p}<0,05)$ entre as faixas etárias avaliadas (Gráfico 2).

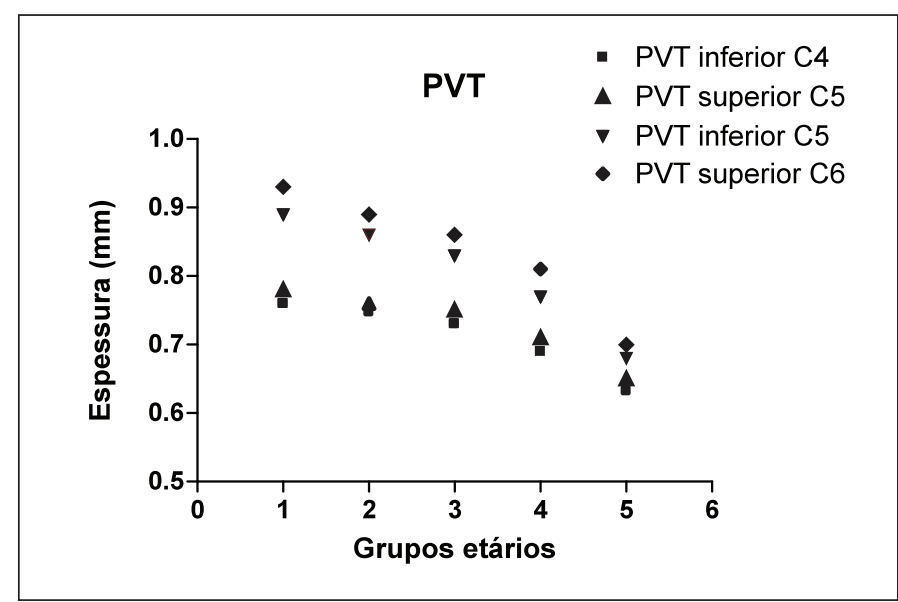

Gráfico 2 - Valores da média da espessura da PVT nos diferentes níveis estudados e nas diferentes faixas etárias. Observar a correlação entre a espessura da PVT adjacentes ao mesmo DI.

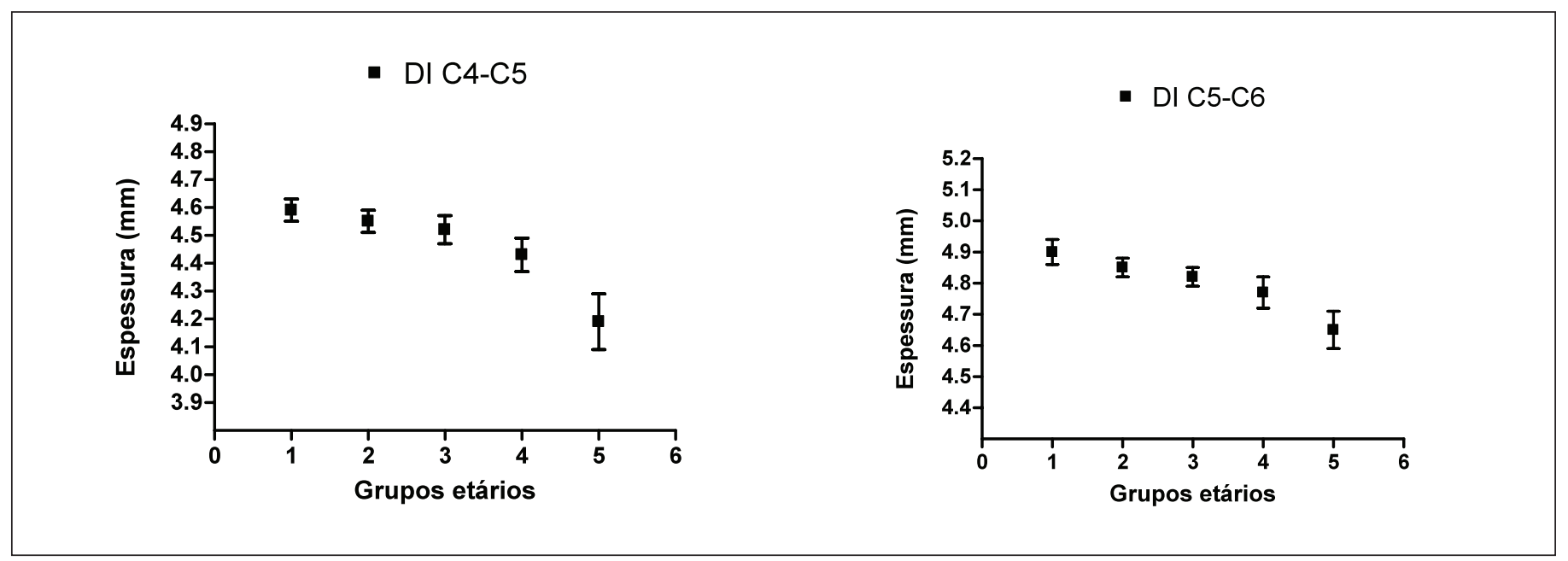

Gráfico 1 - São ilustrados os valores médios e desvio padrão da espessura dos discos intervertebrais C4-C5 e C5-C6.

Tabela 1 - Média dos valores da espessura da PVT e disco intervertebral (C4-C5 e C5-C6) nos segmentos vertebrais estudados.

\begin{tabular}{|c|c|c|c|c|c|c|}
\hline \multirow[b]{2}{*}{ Grupo etário } & \multicolumn{3}{|c|}{ Nível C4-C5 } & \multicolumn{3}{|c|}{ Nível C5-C6 } \\
\hline & PVT C4 & DI & PVT C5 & PVT C5 & DI & PVT C6 \\
\hline & (inferior) & & (superior) & (inferior) & & (superior) \\
\hline 1 & $0,76+0,03$ & $4,33+0,20$ & $0,78+0,02$ & $0,89+0,02$ & $4,65+0,09$ & $0,93+0,02$ \\
\hline 4 & $0,69+0,03$ & $4,23+0,15$ & $0,71+0,03$ & $0,77+0,03$ & $4,53+0,07$ & $0,81+0,03$ \\
\hline 5 & $0,64+0,02$ & $4,08+0,10$ & $0,65+0,02$ & $0,68+0,02$ & $4,41+0,05$ & $0,70+0,07$ \\
\hline Total & $0,71+0,04$ & $4,24+0,08$ & $0,73+0,08$ & $0,80+0,07$ & $4,56+0,08$ & $0,83+0,07$ \\
\hline
\end{tabular}


A espessura das placas vertebrais terminais adjacentes ao DI C4-C5 e C5-C6 não apresentaram diferença estatística $(p>0,05)$ em todos os grupos estudados. No entanto, foi observado diferença estatística entre a altura da PVT distal e proximal de C5. Desse modo, as placas vertebrais adjacentes ao mesmo DI não apresentaram diferença de espessura, embora pertencessem a vértebras distintas. Enquanto que as PVT pertencentes a mesma vértebra mas em contato com DI distintos, apresentaram diferença quanto à espessura.

O coeficiente de correlação entre a altura do DI e a altura da PVT adjacente permaneceu constante nas diferentes faixas etárias, conforme tabela 2 . A redução da altura do DI com o aumento da idade foi acompanhada da redução da altura da PVT, mantendo desse modo a correlação.

Tabela 2 - Valores do coeficiente de correlação entre a PVT e o DI adjacente nos segmentos da coluna cervical relacionados para o estudo. Observar que o coeficiente de correlação foi superior a $80 \%$ em todos os grupos etários.

\begin{tabular}{c|c|c|c|c|c|c|c}
\hline & & & \multicolumn{5}{|c}{ Grupos etários } \\
\hline & & & 1 & 2 & 3 & 4 & $\mathbf{5}$ \\
\hline $\begin{array}{c}\text { Coef. } \\
\text { Correlação }\end{array}$ & C4 & média & 0,88 & 0,86 & 0,84 & 0,84 & 0,86 \\
\hline & & DP & 0,02 & 0,03 & 0,04 & 0,02 & 0,05 \\
\hline PVT X DI & & & & & & & \\
\hline & C5 & média & 0,87 & 0,87 & 0,83 & 0,85 & 0,84 \\
\hline & & DP & 0,03 & 0,05 & 0,02 & 0,02 & 0,04 \\
\hline & C5 inf & média & 0,89 & 0,88 & 0,86 & 0,86 & 0,83 \\
\hline & & DP & 0,06 & 0,04 & 0,01 & 0,03 & 0,05 \\
\hline & C6 & média & 0,88 & 0,85 & 0,84 & 0,85 & 0,88 \\
\hline & & DP & 0,05 & 0,06 & 0,02 & 0,02 & 0,02 \\
\hline
\end{tabular}

\section{DISCUSSÃO}

O DI é uma estrutura avascular e a sua nutrição ocorre por meio da difusão dos nutrientes através da $\mathrm{PVT}^{(8)}$. A difusão através da PVT não é uniforme e a região central tem sido considerada o local da PVT no qual a nutrição do DI seria mais crítica e com maior taxa metabólica, tendo sido esse o motivo para a escolha da parte central da PVT e do DI para o estudo.
Os estudos da nutrição e metabolismo do DI tem demonstrado que a zona central da PVT apresenta maior permeabilidade à difusão de pequenas moléculas de soluto, enquanto que a porção lateral é relativamente impermeável $^{(9)}$. Esta permeabilidade é atribuída à presença de maior número de terminações vasculares na região central do osso esponjoso do corpo vertebral adjacente à PVT. Crock et al demonstraram que o principal forma de transporte de pequenas moléculas de soluto se dá através da porção central da PVT através do processo de difusão ${ }^{(10)}$. Entretanto, outros fatores interferem na passagem de solutos através da PVT para o DI.

A composição bioquímica da PVT apresenta importante papel funcional na manutenção da integridade do DI, sendo os proteoglicanos considerados como importantes reguladores do transporte passivo de solutos essenciais para dentro e fora do DI, devido a suas cargas negativas $^{(11)}$. Semelhantemente ao núcleo pulposo, os proteoglicanos constituintes da PVT, além de participarem da retenção de água, conferem carga predominantemente negativa à PVT e ao núcleo pulposo, facilitando a difusão de solutos pequenos de cargas negativas como o oxigênio e a glicose assim como a difusão de solutos de cargas positivas, como o sódio e cálcio. No entanto, moléculas de grande tamanho e cargas negativas apresentam relativa dificuldade de difusão para o núcleo pulposo, como as imunoglobulinas e macromoléculas enzimáticas. Desse modo o número de terminações vasculares no terço central da PVT seriam os dois principais fatores que influenciam a passagem de solutos pela PVT até o núcleo pulposo ${ }^{(6)}$.

Considerando os conceitos atuais acerca da nutrição do DI e da participação da PVT nesse processo, estabelecemos a hipótese de que poderia haver correlação morfológica entre a espessura da PVT e DI devido à íntima relação funcional entre essas estruturas. A hipótese estabelecida foi confirmada na avaliação inicial, tendo sido notada a correlação entre a espessura da PVT e a altura do DI interposto em todos os níveis estudados e em todas as faixas etárias. Foi observado que a espessura da PVT não apresentou correlação na mesma vértebra, mas com o DI sobre a qual a PVT estava em contato. Essa observação adicional corrobora a relação funcional entre PVT e o DI.

Com o aumento da idade, ocorre redução progressiva na espessura do DI, PVT e na permeabilidade da PVT, ocasionando redução gradual da nutrição discal. Progressivas alterações da composição do DI e PVT de macacos com o aumento da idade, foram observadas em 
associação com a diminuição da quantidade de sulfato de glicosaminoglicanos e de sulfato de condroitina tendo como resultado a redução da espessura da PVT e do DI assim como, da permeabilidade de pequenos solutos que nutriam o núcleo pulposo ${ }^{(12)}$. Similares alterações do DI e PVT foram observadas em humanos ${ }^{(13)}$. Nossos resultados confirmaram essa observação e a possível relação entre o DI e PVT, tendo sido observado em nosso material estudado a redução proporcional destas estruturas com a idade.

A hipótese de que a espessura da PVT apresenta correlação com a espessura do DI foi confirmada nesse estudo. Essa correlação tem sido estabelecida do ponto de vista funcional, e com os resultados morfológicos observados pode estabelecer uma nova abordagem para o entendimento dos fenômenos relacionados com a nutrição e degeneração do DI.

\section{REFERÊNCIAS}

1. Melrose J, Smith SM, Appleyard RC, Little CB. Aggrecan, versican and type VI collagen are components of annular translamellar crossbridges in the intervertebral disc. Eur Spine J. 2008;17(2):314-24.

2. Urban JP, Mcmullin JF. Swelling pressure of the lumbar intervertebral discs: Influence of age, spinal level, composition and degeneration. Spine. 1988;13(2)179-87.

3. Ayad S, Weiss JB. Biochemistry of the intervertebral disc. In: Jayson MIV. The lumbar spine and back pain. 3rd ed. Churchill Livingstone: Edinburg; 1987

4. Moore RJ. The vertebral end-plate: what do we know? Eur Spine J. $2000 ; 9(2): 92-6$.

5. Ghosh P. Basic biochemistry of the intervertebral disc and its variation with ageing and degeneration. J Man Med. 1990;5:48-51.

6. Bishop P. Pathophysiology of the intervertebral disc. In: Haldeman S, editor. Principles and practice of chiropractic. 2nd ed. East Norwalk, CT: Appleton and Lange; 1992.

7. Horner HA, Urban JP. Volvo Award Winner in Basic Science Studies:

\section{CONCLUSÃO}

Nos níveis cervicais avaliados observou-se redução progressiva e proporcional da espessura da PVT e do DI adjacente nas diferentes faixas etárias incluídas no estudo. A placa vertebral terminal apresenta correlação morfológica com o disco intervertebral ao qual entra em contato, e não apresenta correlação com a placa vertebral terminal da mesma vértebra.

\section{AGRADECIMENTO}

À Sra. Auristela de Mello Martins, técnica especialista em histopatologia do Departamento de Patologia da Faculdade de Medicina de Ribeirão Preto da Universidade de São Paulo - USO - Ribeirão Preto (SP), Brasil.
Effect of nutrient supply on the viability of cells from the nucleus pulposus of the intervertebral disc. Spine. 2001;26(23):2543-9.

8. Nachemson A, Lewin T, Maroudas A, Freeman MA. In vitro diffusion of dye through the end-plates and the annulus fibrosus of human lumbar intervertebral discs. Acta Orthop Scand. 1970;41(6):589-607;

9. Maroudas A, Stockwell R, Nachemson A, Urban J. Fators involved in the nutrition of the human lumbar intervertebral disc: cellularity and diffusion of glucose in vitro. J Anat. 1975;120(Pt 1):113-30.

10. Crock HV, Yoshizawa $H$. The blood supply of the lumbar vertebral column. Clin Orthop Relat Res. 1976;(115):6-21.

11. Boos N, Weissbach S, Rohrbach H, Weiler C, Spratt KF, Nerlich AG. Classification of age-related changes in lumbar intervertebral discs: 2002 Volvo award in basic science. Spine. 2002;27(23):2631-44.

12. Buckwalter JA. Aging and degeneration of the human intervertebral disc. Spine. 1995;20(11):1307-14.

13. Gruber HE, Hanley EN Jr. Ultrastructure of the human intervertebral disc during aging and degeneration: comparison of surgical and control specimens. Spine. 2002;27(8):798-805. 